\title{
Educação em saúde a partir de círculos de cultura*
}

\author{
Health education based on culture circles \\ Educación en salud basada en círculos de cultura
}

\author{
Estela Maria Leite Meirelles Monteiro', Neiva Francenely Cunha Vieira" \\ 'Universidade Federal de Pernanbuco. Faculdade de Enfermagem Nossa Senhora das Graças. \\ Grupo de Pesquisa em Epistemologia e Fundamentos do Cuidar. Recife, PE. \\ "Universidade Federal do Ceará. Departamento de Enfermagem. \\ Grupo de Pesquisa Família, Ensino, Pesquisa e Extensão. Fortaleza, CE
}

Submissão: 06/09/2009

Aprovação: 02/10/2009

\section{RESUMO}

O estudo tem os objetivos de sistematizar, mediante Círculos de Cultura, uma proposta de (re)construção das ações de educação em saúde Que articule as competências necessárias aos(as) enfermeiros(as) de PSF para uma prática de educação em saúde reflexiva e crítica e realizar uma auto-avaliação dos Círculos. Constitui uma Pesquisa-ação, onde a pesquisadora fundamentada no "Método Paulo Freire" é animadora de debates em oito Círculos de Cultura. Participaram dez enfermeiras. Os Círculos propiciam o exercício da consciência política, indispensável ao processo de "empowerment" do profissional de saúde no exercício de sua competência sociopolítica. A realização de Círculos de Cultura propicia uma relação de cumplicidade entre os profissionais de saúde e os grupos comunitários com as ações de promoção da saúde.

Descritores: Educação em saúde, Promoção da saúde, Programa Saúde da Família; Enfermagem.

\section{ABSTRACT}

This study aims at systematizing with the participants of Culture Circles a proposal of reconstruction of actions of health education that show the necessary competences to the nurses of Family Health Program for a practice in education on critical and reflexive health. It is constituted of a research - action, where the researcher based in "Paulo Freire Method" is encouraging debates in eight Culture Circles having the participation of ten nurses. The Circles give them the training of a political conscience, essential to the process of " empowerment "of the health professional in practicing his socio-politics competence. The proposal of achieving the Culture Circles establish a link of complicity between health professionals and communitarian groups with the actions of health promotion.

Key words: Health education; Health promotion; Family Health Program; Nursing.

\section{RESUMEN}

El estudio tiene como objetivo sistematizar, con las participantes de los Círculos de Cultura, una propuesta de (re)construcción de las acciones de educación en salud Que articule las competencias necesarias a los(as) enfermeros(as) del PSF para una práctica de educación en salud reflexiva y crítica. Constituye una investigación-acción, donde la investigadora fundamentada en el "Método Paulo Freire" es animadora de debates en ocho Círculos de Cultura contando con la participación de diez enfermeras. Los Círculos propician el ejercicio de la conciencia política, indispensable para el proceso de "empowerment" del profesional de salud en el ejercicio de su competencia sociopolítica. La propuesta de realización de Círculos de Cultura fundamenta una relación de complicidad entre los profesionales de salud y los grupos comunitarios con las acciones de promoción de la salud.

Descriptores: Educación en salud; Promoción de la salud; Programa Salud de la Familia; Enfermería.

Trabalho obteve o primeiro lugar com o Prêmio Laís Netto dos Reis, da Escola de Enfermagem Ana Nery -UFRJ, durante o $60^{\circ}$ Congresso Brasileiro de Enfermagem (CBEn) realizado de 03 a 06 de novembro de 2008 - Minascentro - Belo Horizonte - MG

AUTOR CORRESPONDENTE Estela Maria Leite Meirelles Monteiro. Universidade de Pernambuco, Faculdade de Enfermagem Nossa Senhora das Graças. Rua Arnóbio Marques, 310. Santo Amaro. CEP 50100-130. Recife, PE. E-mail: estelaf2003@yahoo.com.br 


\section{INTRODUÇÃO}

A municipalização da saúde constituiu elemento marcante para implantação dos Programas de Saúde da Família, objetivando promover um acesso da comunidade aos serviços de saúde, de modo a reorientar as ações com enfoque na promoção à saúde buscando estabelecer uma articulação entre as demais instâncias de atenção à saúde, possibilitando encaminhamentos necessários a uma assistência Que atenda, entre outros, ao princípio da integralidade.

O Programa Saúde da Família surge no contexto nacional a partir dos êxitos e dificuldades de modelos anteriores de organização da atenção básica, dentre estes a Ação Programática em Saúde ${ }^{(1)}$, o modelo em Defesa da Vida, os sistemas locais de saúde (SILOS), os distritos sanitários ${ }^{(2)}$, reportando-se, ainda, no plano internacional, às experiências dos modelos de Saúde da Família, particularmente de Cuba e Canadá(3).

Vale ressaltar como alicerce para a reorientação das políticas públicas de saúde, o moderno movimento de promoção da saúde, Que surge no Canadá em 1974 e é propagado para o mundo com a primeira Conferência Internacional sobre o tema em Ottawa no ano de 1986. Como fruto da conferência foi elaborado a Carta de Ottawa enfatizando Que as ações comunitárias serão efetivas se for garantida a participação popular na delimitação de prioridades, na tomada de decisões e na definição e implementação de estratégias para alcançar um melhor nível de saúde. Para tanto, o documento resgata a dimensão da educação em saúde, com a idéia de "empowerment", ou seja, o processo de aQuisição de poder técnico e consciência política para atuar em prol de sua saúde ${ }^{(4)}$.

Como estratégia de promoção à saúde, a práxis de Educação em Saúde, nesta conscientização individual e coletiva de responsabilidades e de direitos, deve eleger metodologias de ensino Que conduzam a uma transformação dos indivíduos socialmente inseridos no mundo, ampliando sua capacidade de compreensão da complexidade dos determinantes de ser saudável ${ }^{(5)}$.

É imprescindível, portanto construir práticas educativas comprometidas com os princípios de cidadania e democracia, e Que leve o indivíduo a sua autonomia e emancipação como sujeito histórico e social capaz de propor e opinar nas decisões de saúde, evidenciando o compromisso social com a melhoria do estado de saúde da população(6).

O exercício de uma prática educativa crítica constitui uma forma de intervenção no mundo, comprometida com o princípio de democracia Que rejeita Qualeuer forma de discriminação, dominação e integra uma atitude de inovação e renovação, na crença de Que é possível mudar $^{(7)}$.

A abordagem de ensino do Círculo de Cultura de Paulo Freire ${ }^{(8)}$, constitui uma idéia que substitui a de 'turma de alunos' ou de 'sala de aula'. A escolha por desenvolver um Círculo de Cultura, visa ensejar uma vivência participativa com ênfase no diálogo, campo profícuo para a reflexão-ação na elaboração coletiva de uma proposta sistematizada para uma educação em saúde emancipatória.

A denominação de Círculo culmina porQue todos estão à volta de uma equipe de trabalho, com um animador de debates Que participa de uma atividade comum em Que todos se ensinam e aprendem, ao mesmo tempo. A maior Qualidade desse grupo é a participação em todos os momentos do diálogo, Que é o seu único método de estudo nos círculos. É de cultura, porque os círculos extrapolam o aprendizado individual, produzindo também modos próprios e renovados, solidários e coletivos de pensar ${ }^{(9)}$.

O desenvolvimento do trabalho com aplicação do Círculo de Cultura requer que o animador(a) esteja atento para o Que se fala. As falas, as conversas, as frases, entrevistas, discussões, dentro ou fora do círculo, tudo está carregado dos temas da comunidade, seus assuntos, sua vida ${ }^{(10)}$.

Tomando por princípio norteador o delineamento do "Método Paulo Freire"(I I), o desenvolvimento do Círculo de Cultura consiste de três momentos: a) a investigação temática, pela Qual os componentes do círculo e o animador buscam, no universo vocabular dos participantes e da sociedade onde eles(as) vivem, as palavras e temas centrais de suas biografias; b) a tematização, mediante a Qual eles(as) codificam e decodificam esses temas; ambos buscam o seu significado social, tomando assim consciência do mundo vivido; e c) a problematização, por meio de Que eles(as) buscam superar a primeira visão mágica por uma visão crítica, partindo para a transformação do contexto vivido.

Constituindo uma estratégia da educação libertadora, o Círculo de Cultura é um lugar onde todos têm a palavra, onde todos lêem e escrevem o mundo. É um espaço de trabalho, pesQuisa, exposição de práticas, dinâmicas, vivências Que possibilitam a elaboração coletiva do conhecimento ${ }^{(12)}$.

A partir do entendimento das ações de Educação em Saúde, como proposta inovadora, articulada entre a concepção da realidade do contexto de saúde e a busca de possibilidades de atitudes geradoras de mudanças, este estudo propõe aos(as) enfermeiros(as), Que atuam em Programa Saúde da Família (PSF) na cidade do Recife, a aplicação de uma metodologia participativa por meio do Círculo de Cultura, inspirado no método de alfabetização de Paulo Freire. A realização dos Círculos de Cultura visa a ensejar uma vivência, entre os(as) enfermeiros(as), de uma proposta de intervenção educativa Que valorize a experiência do grupo e promova sua participação na construção do conhecimento coletivo. Espero, desta forma, contribuir para o desenvolvimento de competências e habilidades desses profissionais, como educadores(as) em saúde, numa perspectiva crítico-reflexiva.

O Que me proponho é a vivência de uma experiência pedagógica e a aQuisição de uma nova técnica para a solução de problemas vitais, com o manejo de Círculos de Cultura, como um instrumento de autonomia. Esta proposta de Paulo Freire pode ser vista a partir da interdisciplinaridade de suas dimensões, pois ele não se percebe apenas como cientista e educador, mas também um sujeito social e político. Ele busca, nas ciências (sociais e naturais), elementos para compreender mais cientificamente a realidade e poder intervir de maneira mais eficaz nela. Por isso ele pensa a educação ao mesmo tempo como ato político, como ato de conhecimento e como ato criador $^{(10)}$

Com base na aplicação do Círculo de Cultura como recurso teórico-metodológico para desenvolvimento desta pesquisa, advogo a seguinte tese: a aplicação dos Círculos de Cultura como abordagem metodológica desenvolvida junto as enfermeiras de PSF possibilita a (re)construção de ações de educação em saúde, tornando-as reflexivas e críticas.

Diante do exposto o estudo tem como objetivo sistematizar, 
com as participantes dos Círculos de Cultura, uma proposta de (re)construção das ações de Educação em Saúde Que articule as competências necessárias aos(as) enfermeiros(as) de PSF para uma prática de educação em saúde reflexiva e crítica.

\section{METODOLOGIA}

Constitui uma Pesquisa-ação, na Qual, em consonância com Peruzzo $^{(13)}$, como enfermeira/pesQuisadora e animadora dos Círculos de Cultura, estabeleço uma interação com as enfermeiras, Que atuam em PSF, sujeitos do estudo, determinando a conjugação da investigação com os processos mais amplos da ação educativa e a apropriação coletiva do conhecimento.

$\mathrm{Na}$ opção por este tipo de estudo foi sedimentada a finalidade de propiciar às profissionais perceberem-se e atuarem como sujeitos políticos no desenvolvimento dos Círculos de Cultura. Constitui, assim, a alternativa de investigação Que visa à inclusão social das enfermeiras, como agentes de conhecimento das ações de Educação em Saúde e beneficiárias dos resultados. Na modalidade Qualitativa de pesquisa, a literatura e a experiência de pesquisar em enfermagem tem evidenciado a importância de sua coerência interna, articulando o rigor no emprego do método e a fidedignidade do pesquisador ao seu referencial teórico ${ }^{(14)}$.

O estudo foi realizado no Distrito Sanitário VI, em RecifePernambuco-Brasil, Que abrange o total de oito bairros, com uma população de 368.844 habitantes. A opção por trabalhar com o VI Distrito deu-se por possuir a mais extensa área física e o maior número de equipes. Em virtude da sua extensão, esse Distrito é subdividido em três microrregiões. Por conseguinte, foi constatada a necessidade de optar por uma delimitação, sendo escolhido a microrregião 6.3, correspondente ao bairro do Ibura, Que apresenta as seguintes características: área bastante acidentada, com elevada concentração populacional, predominando o baixo poder aQuisitivo de seus moradores, população dependente em sua totalidade do SUS, já ter havido iniciativas anteriores de implementar ações de Educação Popular em Saúde por interesse da direção do Distrito.

A coleta de dados foi desenvolvida nos meses de fevereiro a maio de 2006, teve a participação de dez enfermeiras Que atuam no cenário do estudo, Que demonstraram interesse pela temática e aceitaram participar nos oito Círculos de Cultura, por se identificarem com as condições, desafios e perspectivas do estudo em todas as suas etapas.

A pesquisa teve a observância da Resolução no. 196/96 Que norteia a pesquisa envolvendo seres humanos ${ }^{(15)}$. Para tanto, foi obtido Certificado de Apresentação para Apreciação Ética (CAAE - 0074.0.097.000-05), como também, anuência formal das participantes do estudo.

O procedimento para coleta de dados nos Círculos de Cultura, ocorreu com registros fotográficos e filmagem dos materiais produzidos pelo grupo participante. A associação da imagem ao som durante a coleta de dados possibilitou a retomada do material em momento imediatamente posterior e sempre Que se fez necessário, auxiliando no exame do corpus de pesquisa, no registro, na descrição e na análise da informação(16).

A organização e o funcionamento dos Círculos de Cultura propõe um trabalho sistematizado desencadeando a possibilidade de uma reflexão individual e uma construção do conhecimento compartilhada e calcada na troca de experiências. Desse modo, com base na investigação temática Que já foi iniciada nos primeiros contatos Que antecederam a realização dos Círculos foi possível lançar outras visões acerca do planejamento, Que, ainda não sendo conclusivo, foi receptivo a flexibilizações.

Para esta outra visão sobre o planejamento prévio, era fundamental, na reflexão crítica dos dados registrados em cada Círculo finalizado, valorizar falas, gestos e expressões e apreender a leitura de mundo das participantes nos vários momentos Que compuseram a realização de cada um dos oito Círculos de Cultura, como: dinâmica de descontração e/ou sensibilização, atividade lúdica para explorar os conhecimentos prévios do grupo (universo vocabular das enfermeiras), problematização (elaborar e discutir); seleção dos textos Que subsidiam uma boa fundamentação, para discussão e reflexão teórico-prática. Para contribuir na codificação e decodificação dos temas geradores As palavras geradoras utilizadas por Paulo Freire na alfabetização de adultos emergiam do contexto do grupo a partir da relação professor-estudante, como elementos Que iriam subsidiar o processo ensino-aprendizagem embasado no diálogo e na troca de conhecimentos. Neste estudo, pela aplicação do Círculo de Cultura envolver profissionais de saúde com grau de instrução superior, foi possível obter temas geradores Que foram problematizados para subsidiar a discussão); e na busca de seu significado social, provocando uma postura ativa do grupo na consciência do mundo vivido.

A ação educativa consistiu na aplicação de oito Círculos de Cultura como processo participativo para potencializar as ações de Educação em Saúde das enfermeiras do PSF. A seeüência dos Círculos de Cultura foi trabalhada a partir das seguintes Questões norteadoras: Círculo I - Como me percebo? Círculo 2 - Como percebo as famílias? Círculo 3 - O Que é educação? Como é ser educador? Círculo 4 - O Que é Círculo de Cultura? Círculo 5 Como aplicar os Círculos de Cultura na educação em saúde? Círculo 6 - Que preciso reforçar ou modificar na minha ação educativa? Círculo 7 - Como planejar os Círculos de Cultura? Círculo 8 Qual a proposta de educação em saúde construída pelo grupo?

Devido à dimensão do estudo Que constituiu resultado de tese iniciada em 2006 e concluída em 2007, neste artigo é apresentada à descrição do oitavo Círculo de Cultura, Que teve como tema gerador: a construção coletiva de uma proposta renovada e sistematizada das intervenções de educação popular em saúde. O tema gerador norteia a investigação sobre o pensar das enfermeiras referido à realidade, e sobre seu atuar em educação em saúde sobre a realidade, Que é sua práxis ${ }^{(10)}$.

Estas atividades caminharam refletindo o referencial técnicocientífico adeuirido pelas enfermeiras em sua formação, e a sua prática junto ao indivíduo/família/grupos em educação na promoção à saúde.

Neste estudo, a descrição e a organização dos resultados constaram de transcrição das informações coletadas com registro de falas na íntegra, ordenadas mediante narração e discussão, segundo a sequência dos Círculos de Cultura realizados, de onde decorreu a análise e interpretação dos resultados, pelo grupo, do significado da experiência e por mim em diálogo com a literatura, com apreciação de fundamentações teóricas consideradas relevantes e enriquecedoras ao estudo crítico do discurso popular ${ }^{(17)}$. 
O objetivo da análise (ou descrição) desta estrutura cognitiva consiste em oferecer a pesQuisadora melhores condições de compreensão, decifração, interpretação, análise e síntese do material Qualitativo produzido na situação investigativa. "A significação do Que ocorre na situação de comunicação estabelecida pela investigação passa pela compreensão e a análise da linguagem em situação"(17).

Após a realização de cada um dos Círculos de Cultura, eu assistia à gravação e analisava os cartazes, desenhos ou modelagens elaboradas pelas participantes da pesquisa naquele encontro, para complementar o diário de campo, no Qual registrei palavras-chaves e observações significativas e, assim, procedi à digitação ordenada dos dados.

Em vez da estrutura lógico-formal, há na abordagem metodológica da pesQuisa-ação muitas características próprias aos processos argumentativos. Esses aspectos argumentativos se encontram: na apresentação dos problemas estudados e das propostas de solução ou explicações apresentadas conjuntamente por pesquisadores(as) e participantes; nas deliberações relativas à escolha dos meios de ação aplicados; nas avaliações dos resultados da pesQuisa e a correspondente ação desencadeada ${ }^{(17)}$.

\section{RESULTADOS E DISCUSSÃO}

\section{Descrição do $8^{\circ}$ Círculo de Cultura}

No encontro do oitavo Círculo de Cultura para a sensibilização inicial, foi realizada uma técnica de animação com a realização voluntária de exercícios de alongamentos para identificar e minimizar áreas de tensão muscular, de forma Que o grupo pudesse sentir-se à vontade.

Com base no objetivo Que fundamentou a realização dos Círculos, no momento de expressão, mobilizei as participantes do estudo para responder ao seguinte Questionamento: Qual a proposta de Educação em Saúde do grupo Que possibilita articular um desempenho das enfermeiras do PSF para uma práxis de educação em saúde reflexiva e crítica?

Assim, cada enfermeira teve a oportunidade de apresentar sua proposta de ação por meio de um planejamento de Círculo de Cultura.

Andorinha expressou seu desejo de trabalhar com grupo de mulheres, colocando-se aberta a consultá-las e ouvi-las em seus interesses e prioridades Quanto aos temas a serem discutidos. No seu planejamento, propôs a realização de técnicas de apresentação e descontração do grupo. No conteúdo, considerou a possibilidade da discussão sobre sexualidade, Questões de gênero, educação dos filhos, entre outras, partindo de uma visão integral da mulher e não apenas dos aspectos biológicos com enfoque na doença. No objetivo apresentado, expôs a intenção de estimular a participação e expressão das mulheres, contribuindo assim para sua auto-estima, troca de experiências e busca de enfrentamentos, tanto para as necessidades pessoais, como para as da comunidade. Destacou, ainda, a necessidade de consultar e ouvir a opinião das mulheres na avaliação da experiência educativa vivenciada, conforme pode ser apreciado na citação de alguns trechos de sua fala.

Minha proposta busca promover a participação de mulheres em todas as etapas da atividade educativa... na descoberta dos seus anseios(...) expressando como gostariam Que o grupo fosse conduzido...

...o objetivo é estimular o grupo a buscar conhecer mais sobre corpo, sexualidade, Questões de gênero, sentimentos(...) temas envolvendo a saúde da mulher...

Eu Queria realizar uma dinâmica em Que as mulheres, com argila ou massa de modelar, pudessem elaborar representações do corpo e aparelho reprodutor da mulher. E ai para ter idéia do conhecimento popular dessas mulheres...

Curió sentiu-se motivada a trabalhar um grupo com enfoeue na saúde mental, por estar sensibilizada pela demanda bastante significativa de clientes/usuários dependentes de medicação controlada (ansiolíticos), além dos portadores de desordens mentais, como é evidenciado na fala.

Desejo formar um grupo com atenção à saúde mental, pois percebo uma demanda muito grande de pessoas da comunidade com distúrbios mentais, como também de dependentes de benzodiazepínicos...

Salientou Que já estabeleceu algumas parcerias com a assistente social do Centro de Apoio Psicossocial (CAPS) e com a presidente do Comitê dos Neuróticos Anônimos, Que reside no bairro. Expressou o interesse em construir um espaço de expressão e reflexão de suas necessidades e modos de estar na família, na comunidade, na sociedade, como também de desenvolvimento criativo das habilidades manuais e intelectuais do grupo.

Nakamura $^{(18)}$ acentua Que as desigualdades socioeconômicas, a incompatibilidade entre os anseios e as possibilidades reais para satisfazê-los, além de influenciar as situações experienciadas de adoecer, são também responsáveis pelas condições de estresse e problemas de saúde mental.

No Círculo de Cultura todos são participantes e, como tal, devem ser percebidos como sujeitos autônomos com potencialidades e jamais como submissos, inferiores ou incapazes. Cabe a animadora propiciar o espaço do diálogo e da escuta com a participação espontânea de todos, respeitando as individualidades e possibilitando a troca das experiências, de modo que a animadora ensina e aprende com o grupo.

Foi sugerido pelo grupo Que os participantes fossem consultados para nomear o grupo Que estava se formando com uma identidade própria Que não se limitasse ao enfoQue da doença ou do estigma dos distúrbios mentais.

Sabiá apresentou sua proposta de formação de Círculo de Cultura com adolescentes.

...eu e as agentes comunitários de saúde estamos trabalhando com um grupo de adolescentes em encontros Quinzenais nas Quartas-feiras à tarde na Associação de Moradores...

...os conhecimentos com os Círculos de Cultura estou utilizando com os adolescentes...

...estou realizando dinâmicas e atividades de criação e demonstração com a participação dos jovens, valorizando suas 
falas e garantindo um espaço de escuta e respeito na construção do conhecimento...

Ela ressaltou Que, dentre os temas solicitados, eles demonstraram muito interesse em saber sobre métodos contraceptivos, pela precocidade com Que iniciam suas atividades sexuais.

Patativa sempre conversava sobre sua preocupação com as gestantes de sua comunidade.

...identifieuei muitos casos de gravidez na adolescência, além de casos de gravidez de alto risco, o que muito me preocupa pela necessidade de estabelecer um encaminhamento seguro...

...percebi que em muitos casos algumas das gestantes que encaminhava ainda continuavam mantendo seu acompanhamento comigo pelo estabelecimento de uma relação de confiança...

Assim, ao planejar seu Círculo de Cultura, escolheu o grupo de gestantes, a ser realizado na capela do bairro, visto Que a Unidade Básica de Saúde fica um pouco distante e em área mais elevada. Ela destacou o planejamento familiar e o aleitamento como temas de interesse do grupo. Neste sentido, ressalto a apreciação do tema como descoberta de possibilidades, de caminhos, respeitando as escolhas, sem impor uma conduta preestabelecida, de modo verticalizado.

Bem-te-vi, a exemplo de Patativa, também apresentou uma proposta para formar grupo com gestantes. Identificando a dificuldade de espaço físico na própria unidade onde atuam três equipes, articulou a possibilidade de utilizar a estrutura física da Associação dos Moradores, mesmo reconhecendo não possuir as condições mais adequadas.

...junto com as agentes comunitárias de saúde (ACS) Que desejarem participar, iremos inicialmente trabalhar com um grupo de gestantes...

...iremos consultar as gestantes sobre os temas de seu interesse(...) entretanto desde já tenho convicção que a temática o aleitamento materno será abordada com uma "nova" proposta metodológica não como um conhecimento pronto a ser incutido ao grupo, mas através de um diálogo aberto às inquietações e possibilidades de estabelecer uma relação mãe-filho o mais saudável possível...

Com vistas a subsidiar o acesso a um saber científico contextualizado com o saber peculiar às participantes dos Círculos, foi vivenciado um momento de leitura crítica e reflexiva sobre um texto fotocopiado, intitulado, Sistematização ${ }^{(19)}$. O texto foi selecionado por exibir um conteúdo objetivo e de linguagem clara, apresentando inclusive ilustrações Que aproximam o conteúdo trabalhado com situações do cotidiano, além de constituir um material bastante atualizado, apesar do ano de publicação.

Para Falkembach ${ }^{(19)}$, a sistematização é um processo coletivo de produção do conhecimento Que acontece a partir de práticas sociais concretas. E destaca Que, mesmo Que os atores da prática de Educação em Saúde tenham diferentes visões de mundo, a partir do respeito a essa diversidade, é possível dialogar, expor pensamentos e aproximar as visões e chegar a pontos comuns no entendimento e encaminhamento das práticas desenvolvidas, tendo como produção resultante uma unidade sem uniformizar.

No momento de síntese do conhecimento elaborado pelas enfermeiras do PSF participantes do estudo, foi apresentado a seguinte proposta de (re)construção das ações de Educação em Saúde por intermédio dos Círculos de Cultura:

- Os profissionais necessitam estar sensibilizados Quanto à importância do trabalho com grupos.

- Os profissionais necessitam vivenciar a abordagem de ensino Que fundamenta uma abordagem participativa e libertária para melhor apreensão dos limites e possibilidades de sua aplicação no cenário da Educação em Saúde.

- A abordagem tradicional de ensino encontra-se muito enraizada ao longo da formação dos profissionais de saúde, conduzindo a uma automação na sua reprodução nas práticas educativas em saúde, conduzindo a oferta de receitas e imposições de condutas de saúde desarticuladas das expectativas e interesses da comunidade.

- O Círculo de Cultura facilitou tanto a sensibilização do profissional como também novas perspectivas sobre as ações de Educação em Saúde.

- A proposta da realização de Círculos de Cultura passa pela conquista de espaços de escuta, diálogo, participação de todos, troca de experiências, valorização do saber popular, desenvolvimento do pensamento crítico e reflexivo, contextualização do conteúdo, respeito à autonomia dos sujeitos históricos, possibilidades de caminhos e de transformação da realidade.

- A proposta da realização de Círculos de Cultura requer que o profissional de saúde atue não como professor, "dono do saber", mas como animador (a), Que acredita nas potencialidades do grupo, Que não se limita a ensinar, mas também tem interesse de aprender com o grupo, Que é capaz de se encantar com as conQuistas, avanços e descobertas do grupo. É essencial o autoconhecimento, para identificar suas, limitações, valores e preconceitos, para coibir uma atuação de inclusão, descobertas, respeito às diferenças, aprendizagem, humanização, socialização, superação, de possibilidades.

- É necessário Que, ao iniciar o trabalho educativo, cada profissional tenha a liberdade de identificar qual grupo lhe desperta o interesse, seja pelas suas necessidades, seja por sua proximidade, Que lhe traga um significado especial para contribuir no desenvolvimento da experiência de Educação em Saúde numa proposta renovada e criativa.

- É essencial o apoio continuado da equipe central, estando abertos e próximos para facilitar as ações de Educação em Saúde fortalecendo parcerias, disponibilizando material educativo, recursos materiais mínimos, como papel-ofício, lápis-piloto, papel-madeira, lápis-cera, material para modelagem manual.

- Há necessidade do entendimento compartilhado entre equipe de base e equipe central sobre a necessidade de fortalecimento de trabalhos continuados de Educação em Saúde Que considerem os indivíduos em sua integralidade e não apenas orientações fragmentadas definidas de modo verticalizado, em Que onde profissionais e comunidade passam a ser objeto do processo, conduzindo à alienação e não à conscientização popular.

- Valorizar as atividades de recreação e socialização dos grupos 
como estratégia de promoção de auto-estima, aprendizado no estabelecimento de relações sociais, possibilidades de lazer, valorizar atividades culturais e talentos do grupo, oportunidade de compartilhar momentos de descontração e alegria essenciais na promoção da saúde.

- A proposta da realização de Círculos de Cultura requer a participação do grupo, desde a etapa de planejamento, alicerçando o desenvolvimento dos Círculos, como também, na sua avaliação, evidenciando um compromisso mútuo dos profissionais de saúde e do grupo em buscar fortalecer o atendimento das expectativas e necessidades do grupo. Para tanto, o profissional necessita receber as críticas como elemento essencial na pactuação do contínuo crescimento do grupo.

- A proposta da realização de Círculos de Cultura requer dos membros da comunidade uma participação ativa como sujeitos com autonomia para conduzirem sua trajetória histórica, alicerçados no processo de conquista de sua cidadania.

- A proposta da realização de Círculos de Cultura, como abordagem fundamental na prática da Educação em Saúde, vem despertar nos profissionais de saúde uma atitude de in̨uietação e dinamismo ante as Questões de saúde Que tanto afligem as comunidades de baixo poder aquisitivo.

- A proposta da realização de Círculos de Cultura fundamenta uma relação de cumplicidade entre os profissionais de saúde e os grupos comunitários com o entendimento de saúde como produto das reais condições sociais e do compromisso com as ações de promoção da saúde.

Em seguida, solicitei às participantes Que exprimissem a autoavaliação da vivência dos Círculos, sendo exposto como depoimentos:

...eu vou sentir falta. Foi gratificante. Tivemos outros cursos com outros nomes, educação popular, educação em saúde (...) até na especialização, entretanto não foi vivenciado desse modo, com esse eu aprendi bastante. Se esse tivesse sido um módulo da minha especialização, ela já teria valido a pena (Andorinha).

... a metodologia foi completamente diferente contribuindo para Que com a vivencia dos Círculos de Cultura eu compreendesse melhor seus princípios e sua aplicação(...) os conhecimentos foram trabalhados em dose homeopática nos sensibilizando sobre a importância de uma atuação reflexiva em educação em saúde...(Curió).

...antes nos foi imposto para trabalhar com grupo, mas a gente nem sabia como era... (Andorinha).

Já tinha participado de outros cursos para atuarmos em educação popular, inclusive na especialização, porém a experiência desse foi totalmente diferente...(Bem-te-vi).

Foi importante você está sensibilizando e não forçando...
(Patativa).

Achei proveitoso estava começando a trabalhar com adolescentes. Eu tinha grande dificuldade, pois utilizava uma abordagem tradicional. Eles ficavam inquietos, desinteressados. Agora eles participam, discutem, elaboram cartazes, estão motivados (Sabiá).

Quando a prática educativa surge de uma educação transformadora, fundamentada no diálogo e no exercício da consciência crítica, então, as mudanças aparecem como resultado de uma realidade em Que as pessoas envolvidas no processo retornam participativas, a conscientização passa a ter sentido de auto-avaliação, crítica e reflexiva de sua prática de educação em saúde ${ }^{(20)}$.

\section{CONSIDERAÇÕES FINAIS}

O Círculo constituiu um espaço de encontro e descoberta do outro como sujeito, com aspirações, sentimentos e vivências Que precisam ser desveladas a partir do diálogo no grupo, da participação nas discussões, da troca de conhecimentos e experiências. A organização das participantes em formato de círculo, mantendo uma dialogicidade no processo de construção de um saber coletivo, compartilhado e contextualizado, conduziu as enfermeiras a uma viagem interior, no encontro do pessoal, do formal, do informal. O movimento de busca interior promoveu crescimento, pois possibilitou descortinar a superação de limitações e dificuldades pessoais identificadas, Que condicionam o indivíduo a situações de submissão e acomodação diante de sua história de vida e de passividade ante os processos de exclusão em Que os usuários e as famílias da comunidade em Que atuam estão expostos.

O exercício da consciência política é indispensável ao processo de "empowerment" do profissional de saúde no exercício de sua competência sociopolítica. Assim, ele desagrega um modo de pensar e agir compartimentalizado por normas e regras sendo conduzido como massa de manobra para a defesa e interesses outros e suprime uma autonomia integral do(a) enfermeiro(a) diante das diversidades e desafios presentes na realidade brasileira marcada pelas situações de pobreza.

A partir das ineuietações evidenciadas foram gestadas as propostas apresentadas. O universo subjetivo é renovado nas vivências do real; entre desejos e aspirações, o ser humano percebese em um cenário que exige sua atitude diante dos fatos, deixando de ser mero expectador do espetáculo para se assumir como protagonista de sua história de vida.

Percebi, nos momentos de conversa nos Círculos de Cultura, a transformação de mulheres fortalecidas por suas coneuistas de aprendizado no aprendizado daqueles tão próximos, mas Que precisam perceber-se acolhidos e participativos no exercício de sua cidadania em espaços Que lhes sejam significativos. A Educação em Saúde, assim, vem renovar os seres em evolução, caminhantes e perseverantes.

\section{REFERÊNCIAS}

1. Nemes MIB. Ação programática em saúde: recuperação histórica de uma política de programação. In: Schraiber LB, organizador. Programação em saúde hoje. $2^{\mathrm{a}}$ ed. São Paulo: Hucitec; 2000. 
2. Cecílio ICO , organizador. Inventando a mudança na saúde. $8^{\mathrm{a}}$ ed. São Paulo: Hucitec; 2006.

3. Crevelim MA, Peduzzi M. A participação da comunidade na equipe de saúde da família. Como estabelecer um projeto comum entre trabalhadores e usuários? Ciênc Saúde Coletiva 2005; 10(2): 323-31.

4. Buss PM. Uma introdução ao conceito de promoção da saúde. In: Czeresnia D, Freitas CM, organizadores. Promoção da Saúde: conceitos, reflexões, tendências. Rio de Janeiro: Fiocruz; 2003. p. $15-38$

5. Catrib AMF, Pordeus AMI, Ataíde MBC, Albueuereue VLM, Vieira NFC. Promoção da Saúde: saber fazer em construção. In: Barroso GT, Vieira NFC, Varela ZMV, organizadores. Educação em saúde: no contexto da promoção humana. Fortaleza: Demócrito Rocha; 2003. p. 3 I-8.

6. Silva KL, Sena RR, Grillo MJC, Horta NC, Prado PMC. Educação em enfermagem e os desafios para a promoção de saúde. Rev Bras Enferm 2009; 62(1):86-91.

7. Freire P. Pedagogia da autonomia: saberes necessários à prática educativa. $3 \mathrm{I}^{\mathrm{a}}$ ed. Rio de laneiro: Paz e terra; 2005.

8. Brandão CR. O Que é método Paulo Freire. $7^{\text {a }}$ ed. São Paulo: Brasiliense; 2005.

9. Damasceno CF. Educação popular em saúde: intervenção participativa na construção de relações dialógicas entre portadores de diabetes mellitus - adulto (DM2) e profissionais [dissertação]. Fortaleza:Departamento de Enfermagem da Universidade Federal do Ceará; 2003.

10. Freire P. Pedagogia do oprimido. $34^{\mathrm{a}}$ ed. Rio de Janeiro: Paz e Terra; 2002.
11. Lima LO. Método Paulo Freire: processo de aceleração de alfabetização de adultos. In: Lima LO. Tecnologia, educação e democracia. Rio de Janeiro: Civilização Brasileira; 1979. p. $175-$ 6.

12. Freire P. Criando métodos de pesQuisa alternativa: aprendendo a fazê-la melhor através da ação. In: Brandão CR, organizador. Pesquisa participante. São Paulo: Brasiliense; 1999. p. 34-4I .

13. Peruzzo CMK. Observação participante e pesQuisa-ação. In: Duarte I, Barros A, organizadores. Métodos e técnicas de pesQuisa em comunicação. São Paulo: Atlas; 2005. p. 125 145.

14. Boemer MR. A investigação Qualitativa: zelo pelo rigor e pela ética [editorial]. Rev Esc Enferm USP 2006; 40(3).

15. Ministério da Saúde (BR). Conselho Nacional de Saúde. Resolução n 196 de 10 de outubro de 1996. Diretrizes e normas regulamentadoras de pesquisa em seres humanos. Brasília (DF): Ministério da Saúde; 1996.

16. Gil AC. Como elaborar projetos de pesquisa. $4^{\mathrm{a}}$ ed. São Paulo: Atlas; 2002.

17. Thiollent, M. Metodologia da pesQuisa-ação. $14^{\mathrm{a}}$ ed. São Paulo: Cortez; 2005.

18. Nakamura E. Antropologia. In: Assumpção Junior F, Kuczynski E, organizadores. Tratado de psiQuiatria da infância e da adolescência. São Paulo: Atheneu; 2003. p. 59-62.

19. Falkembach EMF. Sistematização. Ijuí: Ed.Unijuí; 1991.

20. Monteiro EMLM, Rolim KMC, Machado MFAS, Moreira RVO. A visão ecológica: uma teia na enfermagem. Rev Bras Enfermagem 2005; 58(3):34I-4 